\title{
Reflets
}

Revue d'intervention sociale et communautaire

\section{Le profilage policier : le syndrome d'une société de classe opprimante}

\section{Lovanie Côté et David Clément}

Volume 22, numéro 1, printemps 2016

URI : https://id.erudit.org/iderudit/1037170ar

DOI : https://doi.org/10.7202/1037170ar

Aller au sommaire du numéro

Éditeur(s)

Reflets, Revue d'intervention sociale et communautaire

ISSN

1203-4576 (imprimé)

1712-8498 (numérique)

Découvrir la revue

Citer cet article

Côté, L. \& Clément, D. (2016). Le profilage policier : le syndrome d'une société de classe opprimante. Reflets, 22(1), 204-215. https://doi.org/10.7202/1037170ar d'utilisation que vous pouvez consulter en ligne.

https://apropos.erudit.org/fr/usagers/politique-dutilisation/ 


\section{Le profilage policier : le syndrome d'une société de classe opprimante}

\section{Lovanie Côté \\ David Clément}

Association pour la défense des droits sociaux de Gatineau (ADDSGatineau) et militants anticapitalistes

L'objectif de cet article est d'amener une perspective critique de la vision dominante et institutionnelle du concept de profilage policier et de démontrer pourquoi nous considérons non seulement que cette vision est problématique d'un point de vue conceptuel, mais également qu'elle participe activement à légitimer les pratiques de profilage illégal par les corps policiers ${ }^{1}$. En effet, les différents écrits universitaires et institutionnels traitant du profilage social se concentrent surtout sur l'analyse des pratiques de profilage dans un cadre légal et normatif, en tentant d'équilibrer la notion de droits fondamentaux et les principes de sécurité publique. D'une part, cette approche ne remet aucunement en question les intérêts de classe qui sont à la source des pratiques de profilage et, d'autre part, les actions mises en place pour dénoncer le profilage ne sortent pas de l'arène de l'État « de droit » et sont presque exclusivement réalisées en collaboration avec les classes capitalistes, particulièrement avec le bras armé (services de la police) qui travaille pour le maintien de ces classes.

Avant de nous lancer dans le vif du sujet, nous jugeons opportun de préciser que l'analyse proposée ici n'est pas objective, et tel n'en est pas le but, car nous croyons que cette objectivité ne peut exister dans une réflexion sur une problématique qui est fondamentalement idéologique. Nous proposons une analyse qui s'appuie sur notre vision de la société comme militants 
anticapitalistes et prolétaires, ainsi que sur nos expériences individuelles et collectives pour abolir le capitalisme. Il importe donc d'établir, dès le départ, certains aspects fondamentaux de nos réflexions, notamment en ce qui a trait au concept de l'État de droit et à celui de la lutte des classes comme centre névralgique de la plupart de nos problématiques sociales.

L'État de droit est le concept central de notre époque qui détermine si une société peut être considérée comme étant démocratique ou non. Brièvement, ce concept prévoit qu'une société se dote de lois qui définissent les balises morales à l'intérieur desquelles la population doit agir, sous peine de mesures coercitives de la part des institutions de répression de l'État. Pour obtenir le qualificatif " démocratique ", ce concept doit s'appliquer universellement à l'ensemble des membres de la société ainsi qu'aux personnes morales. Ce faisant, la population qui se voit imposer par la violence le respect des lois peut s'attendre à ce que les personnes qui gouvernent respectent à la lettre l'ensemble des textes législatifs, sans jamais outrepasser les pouvoirs qui leur sont conférés par les lois. Il y a lieu d'énoncer deux choses. Nous nous opposons fermement à la relation gouvernants-gouvernés qu'impose cette vision de la société. Elle se trouve à la source même de nos critiques concernant la conception «mainstream » du profilage et les moyens d'action mis de l'avant par une majorité de groupes ou de personnes afin de lutter contre ce phénomène. De plus, nous croyons que les dernières décennies, voire les derniers siècles, ont bien démontré que l'État de droit, tel que défini ici, n'a jamais existé en pratique, alors que les classes dirigeantes et possédantes ont toujours utilisé ce concept à leur propre profit.

L'analyse de classe, comme lunette d'analyse pour comprendre les oppressions et violences au sein de notre société, s'oppose ultimement à l'idée que l'État doit prétendre à l'égalité entre les classes par l'adoption de politiques publiques. Au contraire, l'analyse de classe préconise l'abolition pure et simple des classes sociales. Nous entendons par "lutte des classes » une analyse des différentes relations de pouvoir à l'intérieur de notre société, lesquelles sont axées sur les luttes actives entre des classes sociales ayant des intérêts diamétralement opposés, le tout dans un 
rapport de force dominé par une classe en particulier, soit la classe possédante et dirigeante, qui, en plus de détenir les capitaux et le pouvoir politique, détient le contrôle des forces coercitives organisées et armées de notre société. Nous incluons aussi dans cette analyse une compréhension de l'intersectionnalité de tous les systèmes d'oppression (racisme, sexisme, patriarcat, colonialisme, capacitisme, hétérosexisme, cissexisme, transphobie, impérialisme, etc.). Car, en plus de protéger les intérêts économiques et politiques de la classe dirigeante et possédante, les forces policières jouent un rôle clé dans l'imposition des normes sociales que la classe capitaliste, patriarcale et coloniale veut voir régner.

Dans la littérature, le concept du profilage policier est majoritairement divisé en trois composantes distinctes, soit le profilage social, le profilage racial et le profilage politique. Le profilage social est décrit comme une surveillance et une répression accrues des populations marginalisées à cause de leur condition sociale et économique. Pour ce qui est du profilage racial, le même concept s'applique, mais cette fois en raison de la couleur de la peau ou de l'origine ethnique. Finalement, le profilage politique, qui est le plus récent dans la littérature traitant de profilage, s'appliquerait aux personnes en fonction de leurs appartenances idéologiques. Cette manière de nommer les différents types de profilage s'accompagne nécessairement d'une hiérarchisation de ces concepts, notamment grâce à la reconnaissance, ou non, de ces concepts par les institutions de l'État. En 2008, lorsqu'un policier du Service de police de la Ville de Montréal (SPVM) a tué Freddy Villanueva, le profilage racial a été mis à l'avantscène dans le cadre des interventions publiques qui ont suivi cet évènement (Touzin, 2011). Ce concept a même été dénoncé comme étant partie intégrante des interventions du SPVM dans certains quartiers de la ville de Montréal. À l'extérieur du Québec, notamment aux États-Unis, le profilage racial est fortement dénoncé collectivement par les communautés noires dans un contexte où la violence policière est meurtrière pour tant de jeunes Noirs et où la répression étatique de ces soulèvements est tout aussi violente. Depuis quelques années au Québec, grâce à l'intervention de certains groupes communautaires, populaires et 
universitaires, c'est le concept du profilage social qui a été le plus présent dans les discours, et ce, même au sein des milieux politique et policier. Plusieurs recherches ont été réalisées pour documenter les conséquences de ces pratiques sur les personnes qui les subissent, dont un rapport réalisé en 2009 par la Commission des droits de la personne et des droits de la jeunesse du Québec, qui a été considéré comme étant la reconnaissance officielle de ce phénomène par plusieurs (Campbell et Eid, 2009). À la suite de ces écrits, plusieurs corps policiers ont même affirmé vouloir réduire ce genre de pratiques, notamment pour des raisons d'ordre économique et opérationnel. Par contre, rares sont les recherches qui tentent de déterminer les sources réelles et sociétales du profilage policier, préférant se limiter aux effets immédiats et visibles de ces pratiques.

\section{Le profilage, une arme d'oppression des classes laborieuses}

Le profilage policier, qu'il soit social, racial ou politique, tire ses origines d'un même terreau, soit celui de l'oppression systémique des classes sociales exploitées au profit des classes dirigeantes et possédantes. En effet, les pratiques de profilage systémique ont un lien direct avec la défense et/ou le maintien des intérêts capitalistes des classes supérieures, qu'ils soient économiques, politiques ou sociaux. Historiquement et objectivement, les pratiques policières visant des populations en particulier ont répondu activement aux désirs les plus sombres des classes possédantes et dirigeantes, qui comprenaient et comprennent toujours bien que les personnes exploitées sont en surnombre dans le combat continuel que leur livre la classe exploitante. Le profilage est donc utilisé comme arme d'oppression pour attaquer les conditions quotidiennes de vie des groupes de personnes qui pourraient un jour, et avec raison, se révolter contre le fonctionnement de la société capitaliste qui les exploite. En d'autres mots, le profilage policier est non seulement une pratique systémique visant le contrôle social des personnes subissant la pauvreté, mais aussi une utilisation organisée des 
lois et de la violence étatique à des fins purement politiques et économiques. Ainsi, le profilage policier poursuit un objectif très précis, soit celui d'anéantir, en aval, toutes formes de dissidence réelle en provenance des masses de personnes exploitées. Cette utilisation du bras armé de l'État au profit des gouvernements et des corporations révèle au moins une chose, soit le rôle réel des forces policières au sein des sociétés, c'est-à-dire la répression organisée des populations. C'est justement cet angle d'analyse que nous proposons d'utiliser afin de définir et de comprendre le profilage policier. Sans cet angle d'analyse, le profilage ne restera qu'une fatalité quasi-naturelle à étudier ou à critiquer sur sa forme.

À Gatineau, au Québec, l'adéquation entre les pratiques de profilage policier et la défense des intérêts de la classe possédante est particulièrement visible, notamment dans le quartier de l'île de Hull, un quartier historiquement habité par la classe ouvrière. À l'échelle canadienne, au cours des dernières années, la répression étatique face aux mouvements de contestation populaire qui ne respectent pas les balises "démocratiques » établies par l'État (par exemple durant la grève étudiante au Québec en 2012 et le sommet du G20 à Toronto en 2010) a atteint un niveau si important que même les organisations les plus réformistes ne peuvent plus facilement se cacher derrière le concept de l'État de droit. Il est évident que ce dernier n'existe pas actuellement, même si plusieurs tentent encore d'y croire. En présentant brièvement les éléments clés de ces deux problématiques distinctes, nous tenterons d'établir un lien non seulement entre le profilage et les intérêts de classe, mais aussi entre la collaboration des groupes communautaires avec l'État et la légitimation des pratiques de profilage.

\section{L'exemple de l'île de Hull}

Sur le territoire particulier de l'île de Hull, où la grande pauvreté et l'extrême richesse se côtoient quotidiennement, la présence policière est violente et s'exerce spécifiquement dans le but de "nettoyer " un quartier que laVille de Gatineau et le secteur privé veulent « revitaliser ». Pour ce faire, les forces de l'ordre travaillent 
littéralement à expulser les populations historiquement présentes dans ce quartier afin de laisser la place aux entrepreneurs qui désirent y ériger des tours de condominiums pouvant accueillir des populations mieux nanties dans un quartier "purifié " de sa faune historique. L'Île de Hull étant considérée à tort par les classes dominantes comme un quartier "à risque ", les effectifs du Service de police de la Ville de Gatineau (SPVG) sont omniprésents et ciblent une diversité impressionnante de personnes qui possèdent cependant une caractéristique commune, soit de faire partie de la même classe sociale. Cette répression organisée, qui vise à desservir les intérêts économiques de la classe dominante, se traduit concrètement par des émissions massives de constats d'infraction, des arrestations arbitraires, des passages à tabac dans des ruelles sombres ou, encore, une surveillance ciblée qui rendrait n'importe qui complètement paranoïaque. L'Association pour la défense des droits sociaux de Gatineau (ADDS-Gatineau) avait d'ailleurs confirmé l'augmentation flagrante des pratiques de profilage liées à l'émission de constats d'infraction entre 2008 et 2012, notamment sur l'Île de Hull, en dévoilant les résultats d'une analyse produite à partir des documents du SPVG et de la Ville de Gatineau, qui démontrait notamment une augmentation de $57 \%$ du nombre de constats d'infraction émis en vertu du règlement municipal sur "la paix publique et le bon ordre " au cours de cette période (ADDS, s.d.). Bien que ces pratiques soient connues et vécues par le milieu communautaire, elles ne sont pas reconnues officiellement par les institutions de l'État (à l'échelon municipal ou provincial). C'est pourquoi les différentes stratégies de collaboration mises de l'avant par les milieux communautaire et universitaire doivent de facto éliminer de leur vocabulaire les mots " profilages systémiques » et "violences étatiques organisées » afin d'être entendues par les institutions de l'État.

Ainsi, les moyens utilisés par les actrices et acteurs communautaires impliquent souvent la collaboration avec la police ou d'autres représentants de l'État, allant de " pourparlers » sur des cas précis à des formations avec le SPVG, notamment lors des périodes intensives de profilage. Ce faisant, une légitimité de ces pratiques s'installe, alors que seuls les évènements plus spectaculaires sont 
discutés et dénoncés afin non pas de critiquer violemment le profilage en soi mais bien de dénoncer des évènements qui "dépassent les bornes ". Pourtant, si borne il doit y avoir, c'est que le profilage policier est illégal.

Encore une fois, dans une analyse de classe, il importe de choisir son côté. Être neutre constitue en soi une acceptation tacite de cette oppression systémique des classes pauvres par les classes riches, ce qui se traduit notamment par la marginalisation et l'insécurisation des personnes qui désirent lutter réellement et radicalement contre ce système oppressif. Malheureusement, le milieu communautaire, bien qu'il aime vanter les mérites de l'éducation populaire - éducation politique par et pour le peuple dans une vision de libération des classes opprimées - ne sert souvent qu'à solidifier les concepts oppressifs qui sous-tendent les pratiques de profilage, notamment en confirmant les peurs partagées par plusieurs personnes face à la répression de l'État. Au lieu de déconstruire ces peurs au profit de l'action collective des masses, hors des cadres imposés par l'État, le milieu communautaire les entretient en mettant lui-même des balises mensongères dans lesquelles ses actions doivent s'accomplir. Mentionnons par exemple les barrières à la mobilisation qui ont été créées dans les dernières années au sein du milieu en ce qui concerne le respect des lois anti-manifestation et la collaboration avec les forces policières. Il en va de même lorsqu'il est question de profilage social ou de profilage en général. Il nous apparait extrêmement problématique, lorsque nous nous unissons pour dénoncer un acte de profilage, qu'à la première occasion de mobilisation sociale, certains groupes changent leur fusil d'épaule et fassent pression sur leurs alliés pour collaborer avec les forces policières ou pour respecter des lois et règlements oppressifs qui visent à encadrer l'expression de la colère populaire. Il s'agit d'un problème que nous constatons depuis quelques années dans la région de l'Outaouais et qui contribue à mettre un frein à la construction d'un rapport de force pour contrer le profilage policier et qui divise les forces au sein même des milieux communautaire et syndical. C'est ainsi qu'en plus d'invisibiliser les racines des pratiques de profilage social et racial, les milieux communautaire, syndical et universitaire 
participent activement à poser des limites de l'action politique «acceptable ", créant ainsi les sources mêmes du profilage politique.

\section{Le piège de la collaboration}

Dans notre société où la novlangue est omniprésente, on nous martèle que la collaboration entre les différents groupes opprimés de la société et l'État oppresseur est nécessaire pour trouver des solutions à des problématiques sociales, économiques et politiques. Cette vision de la société s'appuie sur un concept de neutralité qui cherche à évacuer complètement le concept de rapport de force entre les classes sociales, ce qui amène plusieurs groupes communautaires à se positionner en "zone tampon " entre les forces de l'ordre et les populations visées par le profilage. Par exemple, ils mettent en place des mesures d'atténuation des effets du profilage qui, la plupart du temps, jouent aussi un rôle direct dans la stigmatisation et la surveillance des populations marginalisées. En outre, certains groupes communautaires travaillent activement à faire retirer des constats d'infraction accumulés par des personnes profilées et négocient avec les services de police ou les institutions judiciaires de l'État des processus de "réhabilitation sociale ». Or, ces négociations incluent majoritairement des conditions légales que les personnes profilées doivent respecter, dont celles de ne pas troubler l'ordre public et de participer à des thérapies ou à des programmes de désintoxication. Ce genre de pratiques amène les groupes communautaires à se placer dans une position d'agence de contrôle social face aux personnes profilées et ne remet jamais en question la responsabilité étatique ou de classe; il a comme résultat ultime la légitimation des pratiques de profilage policier ainsi que la neutralisation politique de groupes organisés. Ces groupes pourraient contester les sources profondes du profilage s'ils analysaient la problématique sous un angle qui n'est pas fondamentalement mensonger, soit celui que nous vivons en démocratie et que l'État prévaut afin d'imposer l'équité entre les différentes classes sociales. 
Depuis le printemps 2012, où la brutalité policière et le profilage politique ont atteint des sommets au Québec, dont à Gatineau, la collaboration avec les forces policières est devenue un débat épineux dans l'organisation d'évènements politiques et sociaux au sein des milieux communautaire et syndical. En fait, la collaboration avec la police est devenue une condition préalable à l'implication de la plupart des groupes communautaires et associations syndicales dans les actions politiques. Dans cette pratique de collaboration, il s'agit donc d'un petit noyau de travailleuses et travailleurs communautaires et syndicaux qui prennent des décisions "éclairées " concernant une action politique, tout en réprimant les groupes qui voudraient changer les plans préétablis et autorisés par la police. Les arguments qui justifient cette collaboration sont encore plus inquiétants, car ils consistent à " objectifier » souvent de manière apolitique les personnes subissant des oppressions, c'est-à-dire les personnes victimes de profilage et les personnes en situation de pauvreté et d'exploitation, en les considérant comme étant "vulnérables » et fragiles. Plutôt que de nous voir collectivement comme des citoyennes et citoyens en colère, voulant nous mobiliser contre cette société qui nous exploite et nous opprime, nous voyons un risque que le rapport intervenante/intervenant-cliente/client reste le même dans les actions de dénonciation de profilage et prenne la forme d'un rapport dénonciatrice/ dénonciateurvictime. L'ADDS est l'un des seuls organismes communautaires de la région, sinon le seul, à avoir un mandat, voté en assemblée générale, de non-collaboration avec des institutions étatiques de répression. L'un des points de litige les plus importants réside dans la divulgation du trajet des manifestations aux forces de l'ordre. Encore une fois, la logique de respect de l'État de droit prend toute la place dans l'analyse faite par plusieurs personnes qui préferent se soumettre à des règlements municipaux interdisant les manifestations, plutôt que de combattre directement ces obstacles à la mobilisation. Effectivement, le fait de donner le trajet à la police implique que nous ne laissons pas aux manifestantes et manifestants le pouvoir collectif de décider des différentes actions 
à mener et, surtout, que nous donnons aux forces policières une longueur d'avance dans la répression de nos mouvements.

L'approche socioterritoriale qui émerge en matière de « lutte à la pauvreté " étatique est caractérisée par la concertation entre les différents acteurs et actrices et constitue un autre exemple concret de ce type de collaboration entre les groupes communautaires et les institutions de l'État, le tout dans des lieux où la prédominance du rapport de force de l'État ne pourrait être plus évidente (StGermain, 2013). En effet, ce genre de processus de concertation, par lequel la finalité des travaux est définie d'avance par les institutions de l'État et où seuls les détails peuvent être débattus, ne permet jamais d'étudier les sources d'un problème particulier. De plus, les actrices et acteurs sociaux ne sont objectivement que des courroies de transmission des plans économiques et politiques de l'État vers le milieu communautaire qui se place en position de sous-traitance. D'ailleurs, si nous portons une attention particulière à l'ensemble des processus de concertation établis par l'État dans les dernières années, nous nous rendons rapidement compte que ceux-ci n'ont rien de démocratique et, surtout, qu'ils n'offrent aucune place à la contestation... La concertation, c'est un processus dans lequel on doit ultimement s'entendre avec une classe qui a des intérêts diamétralement opposés aux siens. En somme, les groupes formant la "société civile " sont souvent appelés à tresser la corde avec laquelle ils pendront leurs conditions de vie et, malheureusement, ils se réjouissent presque continuellement de pouvoir choisir la couleur de la corde dans ces processus de concertation.

\section{Un appel à la lutte et à la confrontation}

Pour conclure cet article, nous souhaitons soulever des débats de fond sur la manière dont on devrait analyser et, ultimement, combattre le profilage policier. Nous tenons à critiquer fermement l'esprit de collaboration entretenu par une vaste majorité des milieux communautaire et universitaire avec les forces policières et l'État quand il est question de profilage. En effet, comme nous 
avons tenté de le démontrer, cette collaboration avec les forces de répression, bien qu'elle puisse sembler intéressante pour "gérer " au quotidien les actes illégaux de la police et de l'État envers les populations marginalisées, n'est en fait qu'une des nombreuses stratégies mises en œuvre par l'État pour conférer une légitimité populaire à ces pratiques opprimantes et théoriquement illégales. Ce faisant, l'État est en mesure de neutraliser un grand nombre d'actrices et acteurs susceptibles d'effectuer une contestation pertinente, en les amenant à participer à la "gestion " des problèmes causés par le profilage policier, alors que le problème à la base, c'est l'État, ses objectifs et sa police. En somme, après plusieurs tentatives infructueuses de " gérer " les pratiques de profilage afin qu'elles soient plus subtiles ou plus " humaines", tout comme plusieurs le font à l'égard des horreurs du capitalisme, il serait objectivement temps de cesser ce genre de travaux pour essayer, cette fois, la contestation et la confrontation ouverte avec les forces de l'ordre, l'État ainsi que les acteurs économiques qui participent activement aux pratiques de profilage.

Face à la violence organisée de l'État contre des populations opprimées, il importe non pas de ménager la chèvre et le chou, mais de s'organiser afin de résister à ces pratiques qui ne visent strictement qu'à maintenir dans la peur ou en prison des populations qui, un jour, auront envie de goûter à la liberté, la vraie. Là devrait être la mission des groupes populaires et du milieu universitaire : rétablir la vérité sur un mensonge qui perdure depuis trop longtemps et auquel plusieurs actrices et acteurs "progressistes " participent activement ou passivement depuis toujours. Pour rétablir cette vérité, il faudrait en premier lieu que les groupes communautaires cessent de se mentir à eux-mêmes et à la population, en faisant la promotion d'un État de droit qui n'a jamais existé et qui n'est pas la réponse à la problématique qu'est le profilage policier. La réponse viable à ce symptôme d'une société violente et oppressante, c'est la lutte totale des classes opprimées contre les classes dirigeantes et possédantes, sans possibilité de compromis. 


\section{Note}

1 Les opinions exprimées dans le présent article n'engagent que les auteurs.

\section{Bibliographie}

ASSOCIATION POUR LA DÉFENSE DES DROITS SOCIAUX DE GATINEAU (ADDSGatineau) (s. d.). Documents produits par l'ADDS-Gatineau à partir des documents de la Ville de Gatineau et du Service de police de la Ville de Gatineau, réf. du $1^{\text {er }}$ février 2016, https://drive.google.com/folderview?id=0B3VY5DZbROueGRsNkVHOWJLb00\&usp=sharing

CAMPBELL, Christine, et Paul EID (2009). La judiciarisation des personnes itinérantes à Montréal : un profilage social, réf. du 9 janvier 2016, http://www.cdpdj.qc.ca/Publications/itinerance_avis.pdf

ST-GERMAIN, Lise (2013). "Initiatives de lutte contre la pauvreté et intervention socioterritoriale intégrée ", Nouvelles pratiques sociales, Vol. 26, No 1, p. 35-49.

TOUZIN, Caroline (2011, 24 février). "Lilian Villanueva : mon fils est mort à cause du profilage racial ", La Presse, réf. du 11 décembre 2015,

http://www.lapresse.ca/dossiers/villanueva-lenquete/201102/24/01-4373610-lilian-villanuevamon-fils-est-mort-a-cause-du-profilage-racial.php 